\title{
Correction to: Facial expression recognition sensing the complexity of testing samples
}

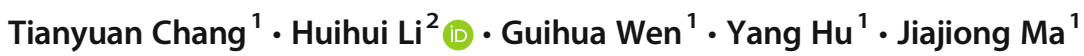

Published online: 27 July 2020

(C) Springer Science+Business Media, LLC, part of Springer Nature 2020

\section{Correction to: Applied Intelligence (2019) 49:4319-4334} https://doi.org/10.1007/s10489-019-01491-8

The original version of this article unfortunately contained a mistake. Graphs c, $\mathrm{d}$ and e are missing in Figure 4. The correct and complete graphs of Figure 4 is shown here.

Publisher's note Springer Nature remains neutral with regard to jurisdictional claims in published maps and institutional affiliations.

The online version of the original article can be found at https://oi.org/ 10.1007/s10489-019-01491-8

Huihui Li

29777562@qq.com

Tianyuan Chang

tianyuan_chang@163.com

Guihua Wen

crghwen@scut.edu.cn

Yang $\mathrm{Hu}$

superhy199148@hotmail.com

Jiajiong Ma

mullma@outlook.com

1 South China University of Technology, Guangzhou 510006, China

2 Guangdong Polytechnic Normal University, Guangzhou 510665, China 


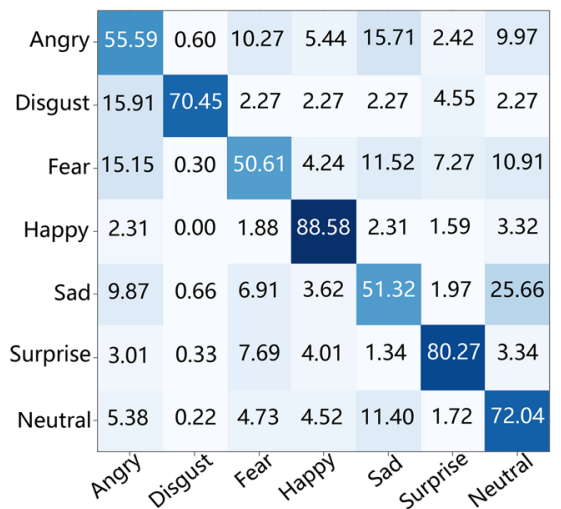

(a) Fer2013_baseline

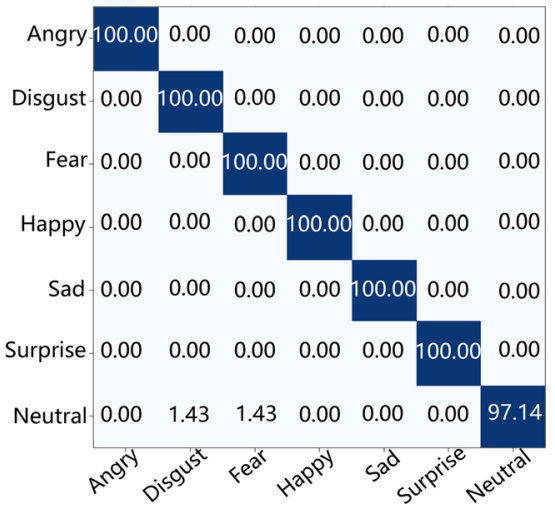

(d) $\mathrm{CK}+\mathrm{CPC}$

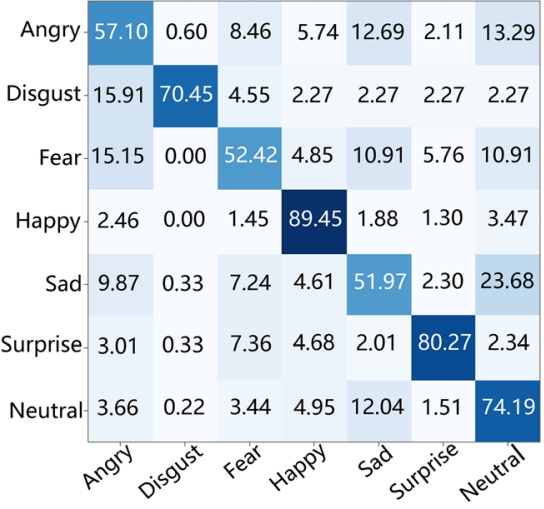

(b) Fer2013_CPC

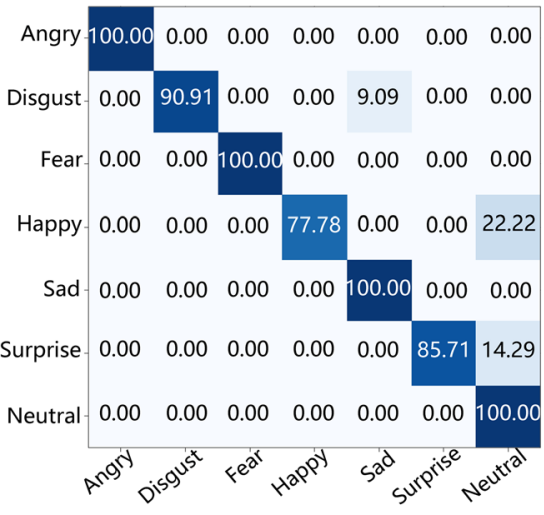

(e) JAFFE_baseline

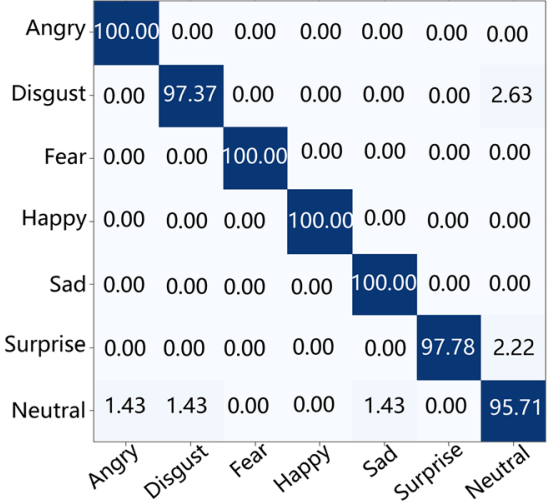

(c) CK+_baseline

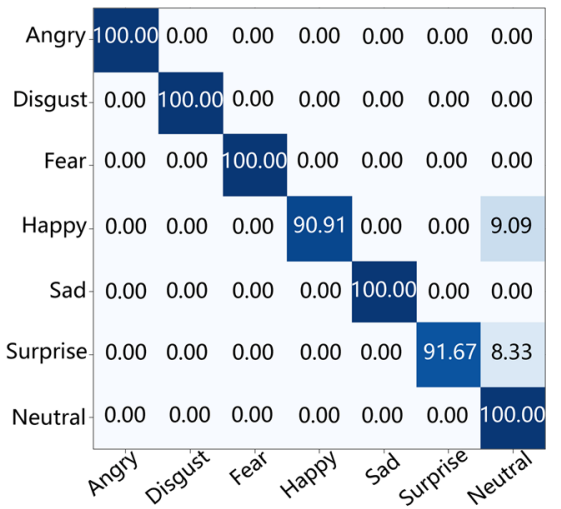

(f) JAFFE_CPC

Fig. 4 Confusion matrices for facial expressions on three datasets. The baseline refers to the case that CNN is taken for feature extraction and Softmax is the classifier. CPC also takes CNN for feature extraction and Softmax as the base classifier. 